\title{
Arteria Lusoria Facilitates Hybrid Management of Aortic Arch Pseudoaneurysm
}

\author{
Simon Bejjani ${ }^{1}$, Nadine Kawkabani ${ }^{2,}$, , Rula Darwish ${ }^{2}$, Omar Boustros $^{1}$, Moussa Abi Ghanem ${ }^{3}$, \\ Bassam Abu Khalil ${ }^{3}$ \\ ${ }^{1}$ Department of Cardiothoracic Surgery, St Georges Hospital-University Medical Center, University of Balamand, Beirut, Lebanon \\ ${ }^{2}$ Department of Cardiac Surgery Anesthesia, St Georges Hospital-University, Medical Center, Beirut, Lebanon \\ ${ }^{3}$ Department of Cardiothoracic Surgery, St Georges Hospital-University Medical Center, Beirut, Lebanon
}

Email address:

nadine_kaoukabani@yahoo.com (N. Kawkabani)

${ }^{*}$ Corresponding author

\section{To cite this article:}

Simon Bejjani, Nadine Kawkabani, Rula Darwish, Omar Boustros, Moussa Abi Ghanem, Bassam Abu Khalil. Arteria Lusoria Facilitates Hybrid Management of Aortic Arch Pseudoaneurysm. International Journal of Cardiovascular and Thoracic Surgery.

Vol. 4, No. 3, 2018, pp. 26-29. doi: 10.11648/j.ijcts.20180403.12

Received: July 24, 2018; Accepted: August 6, 2018; Published: September 1, 2018

\begin{abstract}
Classic open ascending aortic replacement is an effective treatment for Stanford type A aortic dissection However it is associated with a mortality ranging between 15 to $60 \%$. This incidence is even higher in recurrent cases. In these patients, hybrid procedures which combine thoracic endovascular aortic repair (TEVAR) with aortic arch vessel bypasses are successfully adopted. This paper reports a successful hybrid management of aortic arch pseudoaneurysm in a 66 year old female patient known to be hypertensive, diabetic,smoker dyslipidemic on dialysis and who underwent one year previously a surgical ascending aortic repair. Aortic arch vessel bypasses -necessary in this case - were facilitated by the presence of an aberrant right subclavian artery known as Arteria Lusoria- This variant, a rare embryologic anomaly of the aortic arch vessels, described by Hunauld in 1735, was used as an inflow artery to the right common carotid allowing the surgeons to avoid a left to right common carotid artery bypass known to be associated with many complications. Although the hybrid management seems a feasible and safe option especially in high risk patients not eligible for open surgical repair, furher clinical studies and development of new devices dedicated to treat ascending aortic diseases are fundamental to improve outcomes.
\end{abstract}

Keywords: Aortic Arch Pseudoaneurysm, Hybrid Management, Arteria Lusoria

\section{Case Report}

A 66 years old female is admitted to our emergency department with a four days history of chest pain high blood pressure resistant to her medications and a $10 \mathrm{~cm}$ pulsatile midline neck mass covering the sternoclavicular junction and the manubrium.

The patient is known to be hypertensive, diabetic, dyslipidemic and smoker (70py). She has an end stage kidney disease and is on dialysis. She also has an essential thrombocythemia.

To note that the patient developed one year ago a Stanford type A aortic dissection and underwent an emergent ascending aortic replacement with a34 $\mathrm{mm}$ Dacron graft. The surgery was followed by an uneventful postoperative course.
Throughout the first year postoperatively, the patient was admitted three times to the emergency room with chest pain and high blood pressure. CT scan of the chest revealed no new changes and the patient was discharged after controlling her blood pressure.

In this last admission, the patient was stabilized hemodynamically by administering drugs to lower her blood pressure and heart rate. A CT scan of the neck and chest was obtained.

The laboratory results were within normal range except a low hemoglobin $(10 \mathrm{mg} / \mathrm{dl})$ and a high creatinine $(4.1 \mathrm{mg} / \mathrm{dl})$.

The CT scan revealed a pseudoaneurysm of the aortic arch, extravasation of the contrast from the distal anastomosis line of the previous surgery and extension of the dense fluid to the anterior mediastinum and across a defect to the sternum into 
the subcutaneous tissues of the suprasternal region. "figure 12"

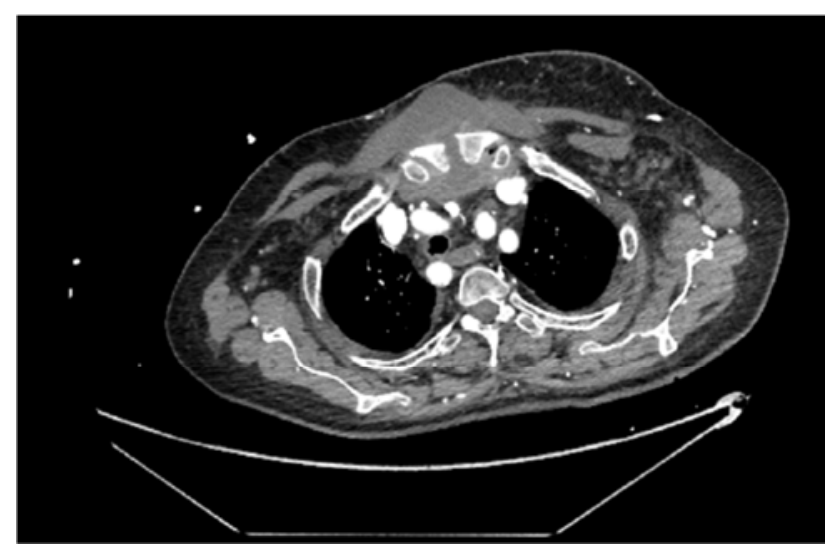

Figure 1. Neck hematoma post aortic dissection and rupture.

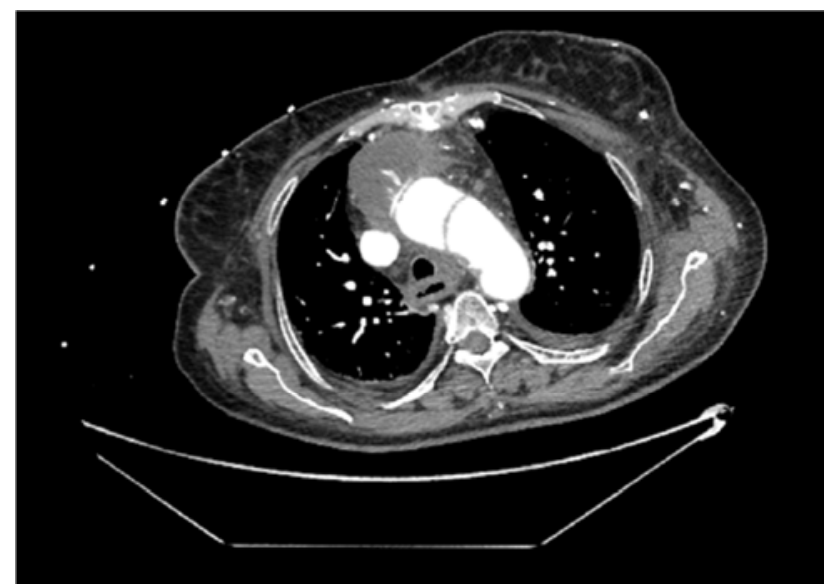

Figure 2. Type A aortic dissection with extravasation of contrast from previous anastomotic line.

The scan revealed also the presence of an arteria lusoria which is an aberrant right subclavian artery.

The patient was admitted to the intensive care unit for vigorous blood pressure and heart rate control.

A multidisciplinary team including cardiac and vascular surgeons, cardiologist, radiologist and anesthesiologist discussed and reviewed the case. Since a surgical replacement of the aortic arch was considered very risky (euroscore 54.7\%), a hybrid management seemed safer and more viable option.

The patient was scheduled few days later for a thoracic endovascular aneurysm repair (TEVAR) combined to a bilateral subclavian to carotid bypasses.

The procedure was performed under general anesthesia Two intravenous lines and a left radial artery cathether were inserted before induction. A central line and a swan ganz were not placed.

After scrubbing and draping, the left common femoral artery was exposed and controlled.

Two separate teams worked simultaneously to perform a left subclavian to left carotid bypass and a right subclavian to right carotid bypass using $9 \mathrm{~mm}$ gortex grafts.

After performing the bypasses. a thoracic endovascular stent graft was inserted through the exposed left femoral artery and inserted across the pseudoaneurysm.

The stent graft was placed proximally inside the previous ascending aortic dacron graft and distally before the insertion of the left subclavian artery. Of note is that the right subclavian artery was spared because it had a low insertion in the proximal descending aorta.

After insertion, the endovascular graft was tested for any endoleaks by a completion arteriography "figure3-4".

At the end of the procedure, the patient was transferred to the coronary surgical unit (CSU). She was extubated the next day after dialysis. The patient had no neurological sequela and had an uneventful postoperative course. She was discharged from hospital one week later.

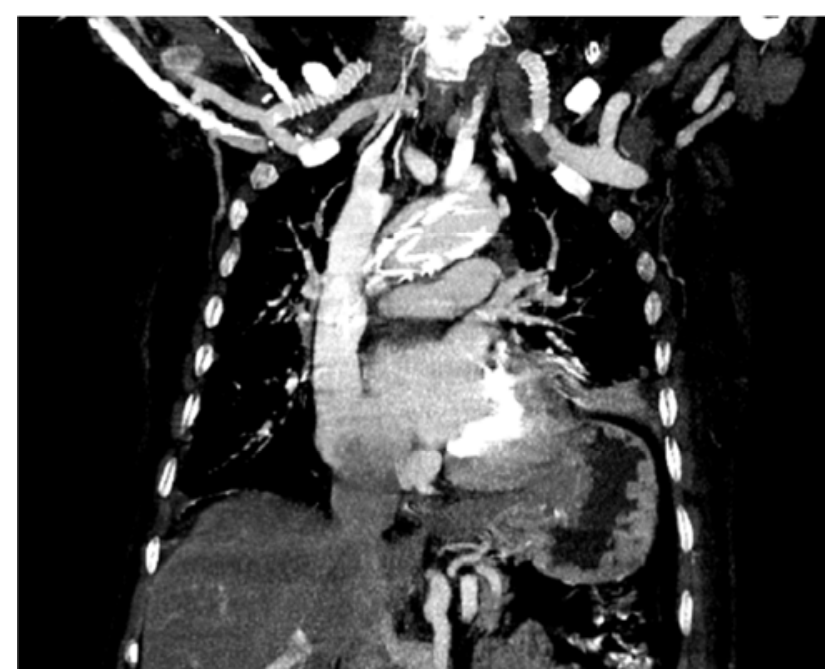

Figure 3. Post operative repair showing the covered stent in the ascending aorta with the two PTFE grafts reaching the carotids.

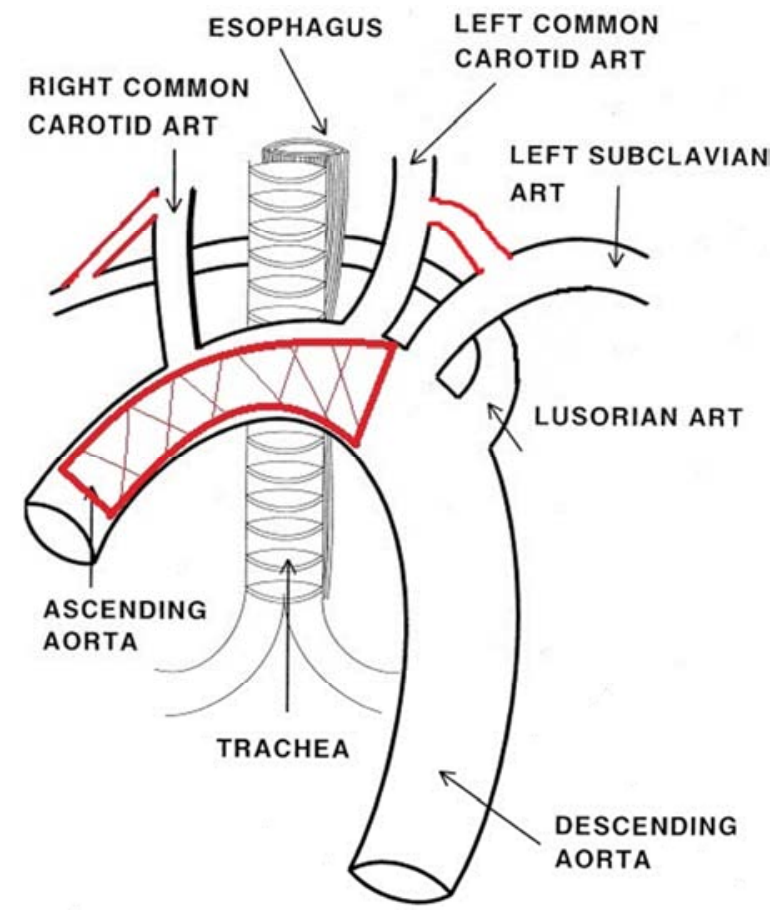

Figure 4. The drawing illustrates the placement of the stent in the aorta and the bilateral subclavian artery to carotid artery bypasses. 


\section{Discussion}

Traditional open aortic replacement is an effective treatment for Stanford type A aortic dissection. However it is associated with a mortality rate ranging from 15 to $60 \%$. This rate is even higher in recurrent cases mainly due to adhesions which render the opening of the chest difficult and hazardous [1].

In such high risk patients, thoracic endovascular aortic repair (TEVAR) of the ascending aorta and aortic arch has been used as an alternative therapy [2]. When necessary the insertion of the stent can be preceded by aortic arch vessels bypasses in order to restore blood supply to the neck and head that can be compromised in some cases. [1]

At the moment, there are only few reports that describes cases of type A aortic dissection treated either with an endovascular technique alone or combined to arterial bypass whenever the blood supply of the brachiocephalic trunk, the right common carotid and the right subclavian artery supply is compromised.

In 2000 Dorros et al reported the first case of type A aortic dissection managed with en endovascular technique through a transseptal approach [3], while Metcalfe et al described a case of endovascular repair of an acute type A dissection using an endograft designed for the ascending aorta introduced through the common femoral artery [5].

Yee et al reported in 2011, 45 cases of type A aortic dissection treated with endovascular stent grafting The entry tear was located at the ascending aorta in 10 cases, the aortic arch in 14 cases and distal aortic arch in 21 cases. The surgical success rate was $97 \%$ and the 90 days mortality rate was $6.7 \%$. [1]

The improvement of imaging has played an important role in facilitating the endovascular technique. In fact the development of multi detector slice CT scan and post processing software has resulted in superior imaging with less artefacts. On the other hand three dimensional reconstitution and virtual endoluminal imaging has been useful to assess the size and location of the intimal tear and to determine the precise length and diameter of the stent graft for successful repair of the dissection. [6]

Transesophageal echocardiography should be also performed in all cases to assess cardiac and aortic valve function as well as an angiography to confirm patency of the supraaortic branches and coronary arteries.

Intravascular ultrasound is also a valuable source in providing supplemental endoluminal information. [5]

Besides imaging, a relative new classification of the aortic dissection "The Dissect" has been particularly useful for endovascular therapy. It encompasses six features:

Duration, Intimal tear location, Size of the aorta, Segmental extent of dissection involvement, Clinical complications (malperfusion...) and Thrombus of the false lumen [7].

In this case, the patient underwent one year ago a classic repair of the ascending aorta following a type A aortic dissection by replacing the dissected part with a $34 \mathrm{~mm}$
Dacron graft. The postoperative course was uneventful. However, throughout the following year she presented three times to the emergency department with chest pain and severe hypertension (200mmhg) despite maximal medical treatment A CT scan of the chest done showed no new changes in the aortic arch nor the descending aorta.

She was admitted lately to the emergency room with chest pain, a large $(10 \mathrm{~cm})$ pulsatile neck mass and high blood pressure (200mmhg). A CT scan of the neck and chest in this last admission revealed an aortic arch pseudoaneurysm at the distal site of the ascending aortic graft.

To note that the patient is known to have an aberrant right subclavian artery also known as Arteria Lusoria which may have predisposed the patient to aortic tear or dissection but facilitated on the other hand the surgical technique.

The Arteria Lusoria is an embryologic anomaly of the aortic arch vessels. This variant has been described first in 1735 by Hunauld. The brachiocephalic trunk is absent and four large arteries rise from the arch of the aorta:Right common carotid artery, left common carotid artery, left subclavian artery and aberrant right subclavian artery known as Arteria Lusoria and which is the most distal left sided origin [8]. The incidence of this variant differs throughout the world. It is in Europe around $0.1 \%$ while it is $0.8 \%$ in the USA. It is more common in female than male subjects. (55.7 vs $45.3 \%$ ).

This embryological variation is also associated with many syndrome such as Down syndrome, Di George syndrome, Edwads syndrome, Tetrallogy of Fallot. The most common vascular anomalies coexisting were trunk bicaroticus, kemmels diverticulum, aneurysm of the Arteria Lusoria at its origin and right sided aortic arch.

Schneider et al noticed that an aortic tear or dissection may occur more frequently during a deceleration trauma when an aberrant right subclavian artery is present. This may be explained by the pathophysiological mechanisms involved in such situations. In fact, abnormalities in the wall of arteries that derived of the fourth arch (proximal part of the right subclavian artery and segment B of the aortic arch) may explain why these arteries are subject of specific anomalies and pathologies. It is important to mention that theses anomalies may be also a risk factor for the development of ostial stenotic lesion of the Arteria Lusoria. [9]

In the case presented in this paper, the patient who is a female subject, had a previous ascending aortic dissection treated by a standard surgical repair. She developed one year post operatively a pseudoaneurysm of the proximal arch with a large $(10 \mathrm{~cm})$ pulsatile neck hematoma originating from the arch and presenting at the sternal notch. This latter finding in addition to expected adhesions prevented the surgeons considering a redo sternotomy.

A multidisciplinary team inlcuding cardiac and vascular surgeons, cardiologist, radiologist and anesthesiologist reviewed the case. Since a redo surgical option was a high risk procedure, they opted for a hybrid surgery that combined the insertion of an endovascular stent graft in the aortic arch with a double arterial bypass from the patent left subclavian 
artery to the left common carotid artery and from the aberrant right subclavian artery (Arteria Lusoria) to the common carotid artery.

The procedure was performed under general anesthesia, The graft was introduced through the left femoral artery. Its placement was preceded by double arterial bypasses. A standard subclavian artery to common carotid artery bypass was performed with a $9 \mathrm{~mm}$ graft.

The presence of the Arteria Lusoria may have predisposed the patient to aortic tear and dissection. However it facilitated tremendously the bypass to the right common carotid artery. In fact it allowed the surgeons to use the aberrant right subclavian artery as the inflow artery for the bypass to the right common carotid. Otherwise a left common carotid to right common carotid artery bypass would have been necessary with all its risks and complications.

\section{Conclusion}

This paper describes a hybrid management of an aortic arch pseudoaneurysm extending to the neck area in a patient who previously had a replacement of her ascending aorta secondary to a type A dissection.. The procedure combined an endovascular repair of the aortic arch pseudoaneurysm with aortic arch vessels bypasses. The surgery was particularly facilitated in this case by the presence of an aberrant right subclavian artery known as arteria lusoria that was used as an inflow artery to the right common carotid. Therefore a carotid- carotid crossover bypass and its complications were avoided

The hybrid management seems a feasible, relatively safe option especially in high risk cases of aortic diseases, however further clinical studies are mandatory.

\section{References}

[1] Ye C, Chang G, Li S, Hu Z, Yao C, Chen W, Li X, Wamg S. Endovascular stent-graft treatment for Stanford type A aortic dissection. Eur j vasc Endovasc surg. 2011; 42:787-794.

[2] Roselli E, Idrees J, Greenberg ROJ, Johnston D, Lytle B. Endovascular stent grafting for ascending aorta repair in high risk patients. J thorac cardiovasc surg. 2015; 149:144-154.

[3] Sobocinsky J O, brien N, Maural B, Bartoli M, Gourffic Y, Sassard T, Midulla M, Koussa M, Vincentelli A, Haulon S. Endovascular approaches to acute aortic type A dissection: A CT based feasability study. Eur j vasc endovasc surg. 2011; 42:442-447.

[4] Ronchey S, Serrao E, Alberti V, Fazzini S, Trimarchi S, Tolenaar J L, Mangialardi N. Endovascular stenting of the ascending aorta for type A aortic dissections in patients at high risk for open surgery. Eur vasc and endovasc surg. 2013; 45:475-480.

[5] Metcalfe M J, Karthikesalingam A, Black S A, LoftusI M, Morgan R, Thompson MM. The first endovascular repair of an acute type A dissection using an endograft designed for the ascending aorta. J vasc surg. 2012; 55:220-222.

[6] Shah A, Khaynezhad A. Thoracic endovascular repair for acute type A aortic dissection: operative technique. Ann cardiothorac surg. 2016; 5 (4):389-396.

[7] Dake M D, Thompson M, Van Sambeek M et al. Dissect: A new mnemonic-based approach for the categorization of aortic dissection. Eur J vasc endovasc surg2013; 46:175-190.

[8] Polgui M, Chrzanowski L, KasprzakJ D, Stefanzyk L, Topol M, Majos A. The Aberrant right subclavian artery (Arteria Lusoria):The Morphological and clinical aspects of one of the most important variations-A systemic study of 141 reports. Scientific world J.2014:1-6.

[9] Shneider J, Baier R, Dinges C, Unger F: Retroesophageal right subclavian artery (lusoria) as origin of traumatic aortic rupture. Eur J Cardio thorac surg. 2007; 32 (2):385-387.

[10] Kirsh M, Soustelle C, HouelRHillion M L, Loisance D Risk factor analysis for proximal and distal reoperations after surgery for acute type A aortic dissection. The journal of thoracic and cardiovasc surg; 123 (2):318-325.

[11] Kolvenbach R R, Karmel R, Pinter L S, Zhu Y, Lin F, Wassijew $\mathrm{S}$ et al. Endovascular management of ascending aortic pathology. J vasc surg. 2011; 53:1431-1437.

[12] Lu Q, Feng J, Zhou J et al. Endovascular repair of ascending aortic dissection:a novel treatment option for patients judged unfit for direct surgical repair J Am coll cardiol. 2013; 61:1917-1924.

[13] Zimpfer D, Czerny M, Kettenbach J, Shoder M, Wolner E, LammerJ et al. Treatment of acute type A dissections with aortic arch involvement. J cardiovasc surg (Torino). 2006; 47:497-502.

[14] Ihnken K, Sze D, Dake M D, Fleischermann D, van der Srarre P, Robbins R. Successful treatment of Stanford type A dissection by placement of a covered stent graft in the ascending aorta. $\mathrm{J}$ thorac cardiovasc surg. 2004; 214:18081810 .

[15] Molz G, Burri B. Aberrant subclavian artery (Arteria Lusoria): sex differences in the prevalence of various forms of the malformation evaluation of 1378 observations virchows archive. Pathological Anatomy and Histology. 1978; 380 (4):303-315.

[16] Nakajima Y, Nishibatake M, Ikeda K, Momma K. Takao A, TeraiM. Abnormal development of fourth aortic arch derivatives in the pathogenesis of tetralogy of Fallot. Pediatric cardiology. 1990; 11 (2):69-71. 